\title{
LETTER
}

\section{Evidence for trans-generational medication in nature}

Thierry Lefèvre, ${ }^{1}$ * Lindsay
Oliver, $^{1}$ Mark D. Hunter ${ }^{2}$ and
Jacobus C. de Roode
${ }^{1}$ Biology Department, Emory
University, 1510 Clifton Road,
Atlanta, GA 30322, USA
${ }^{2}$ Department of Ecology and
Evolutionary Biology, University
of Michigan, 1141 Natural
Sciences Building, 830 North
University, Ann Arbor,
MI 48109-1048, USA
*Correspondence: E-mail:
telefev@emory.edu

Thierry Lefèvre, ${ }^{1 *}$ Lindsay Oliver, ${ }^{1}$ Mark D. Hunter ${ }^{2}$ and Jacobus C. de Roode ${ }^{1}$ ${ }^{1}$ Biology Department, Emory University, 1510 Clifton Road, Atlanta, GA 30322, USA

${ }^{2}$ Department of Ecology and Evolutionary Biology, University of Michigan, 1141 Natural Sciences Building, 830 North University, Ann Arbor, MI 48109-1048, USA telefev@emory.edu

\begin{abstract}
Parasites pose a serious threat to host fitness, and natural selection should favour host traits that reduce infection or disease symptoms. Here, we provide the first evidence of trans-generational medication, in which animals actively use medicine to mitigate disease in their offspring. We studied monarch butterflies and their virulent protozoan parasites, and found that neither caterpillars nor adult butterflies could cure themselves of disease. Instead, adult butterflies preferentially laid their eggs on toxic plants that reduced parasite growth and disease in their offspring caterpillars. It has often been suggested that sick animals may use medication to cure themselves of disease, but evidence for the use of medication in nature has so far been scarce. Our results provide evidence that infected animals may indeed use medicine as a defence against parasites, and that such medication may target an individual's offspring rather than the individual itself.
\end{abstract}

\section{Keywords}

Host-parasite ecology, monarch butterfly, Ophryocystis elektroscirrha, self-medication, tritrophic interactions.

Ecology Letters (2010) 13: 1485-1493

\section{INTRODUCTION}

Parasites are found in all organisms (Windsor 1998), and can dramatically reduce host fitness. Natural selection should therefore strongly favour host protective mechanisms that reduce the risk of infection or minimize parasite-induced fitness loss (Combes 2001). Of the many protective mechanisms that hosts have evolved (Moore 2002; Rolff \& Siva-Jothy 2003; Råberg et al. 2007), the use of medication (Clayton \& Wolfe 1993; Lozano 1998) is perhaps the most intriguing: the idea of animals medicating themselves is not only intuitively appealing, but may also help us in finding ways to combat our own pathogens (Huffman 2003).

Studies of medication in animals have traditionally focused on 'self'-medication, through which animals use third species or compounds to prevent infection of or reduce parasite growth in their own bodies. Self-medication can come in two distinct forms. Prophylaxis is the prevention or reduction of the likelihood of infection and can be displayed by uninfected and infected individuals alike (Christe et al. 2003; Chapuisat et al. 2007; Castella et al. 2008). In contrast, therapeutic self-medication is the curative use of anti-parasitic substances by already infected individuals to fight or eliminate the pathogen (Lozano 1998). Evidence for therapeutic self-medication is still scarce and mostly based on observational field studies of vertebrates
(Wrangham \& Nishida 1983; Huffman \& Seifu 1989; Huffman 2003). Where experimental approaches have been performed, they have often used artificial anti-parasitic diets that do not occur in the wild (Lisonbee et al. 2009). Recent studies have therefore argued that manipulative experiments are badly needed to rigorously test whether animals can cure themselves of disease (Lozano 1998; Hutchings et al. 2003; Singer et al. 2009) and that herbivorous insects and their parasites are suitable systems because of the intricate relationships between insect, parasite and plant chemicals (Lee et al. 2006; Povey et al. 2009; Singer et al. 2009).

Insect-parasite-plant systems also have the added benefit that therapeutic medication behaviours can be studied more broadly in the context of inclusive fitness (Hamilton 1964; Grafen 1984). So far, convincing studies of therapeutic medication have focused on individuals curing themselves (Lee et al. 2006; Povey et al. 2009; Singer et al. 2009), but inclusive fitness theory predicts that individuals can also increase their fitness by mitigating disease in their relatives (Hart 1990). Although such behaviour may be referred to as 'self-medication' from the gene's point of view, we here use 'trans-generational medication' to avoid confusion with behaviours that affect the organism itself. In the case of herbivorous insects, oviposition choices of adults crucially affect larval fitness (Thompson 1988; Gripenberg et al. 2010), and it is conceivable that adults, infected with 
vertically transmitted parasites, can mitigate disease in their offspring by preferentially laying eggs on anti-parasitic host plants.

Here, we use monarch butterflies (Danaus plexippus) to test for the existence of therapeutic self- and trans-generational medication. Our study hinges on the demonstration of four requirements that have to be met to conclude that a behaviour is an adaptation that allows infected hosts to fight their parasites. First, a therapeutic behaviour is specifically displayed by infected individuals only. Second, the behaviour has to result in an alleviation of the fitness loss of the infected individual or its offspring; thus, demonstrating that a behaviour reduces parasite burdens, without showing a reduction in disease symptoms (Råberg et al. 2007), does not provide evidence for therapeutic medication. Third, the behaviour should come at a cost to uninfected individuals; otherwise the behaviour would be displayed by both infected and uninfected animals (Singer et al. 2009). Fourth, the parasite should not benefit from the behaviour; if it does, the behaviour may be a parasitic manipulation (Lefèvre et al. 2009) rather than a host protective response.

Monarch butterflies are commonly infected with the protozoan parasite Ophryocystis elektroscirrha (Leong et al. 1997; Altizer et al. 2000). This host-parasite interaction provides an ideal system to study medication for two main reasons. First, O. elektroscirrha has strong detrimental effects on its host. Parasite infections occur when caterpillars ingest spores that are passively scattered onto eggs or host plant leaves by female adult butterflies during oviposition (McLaughlin \& Myers 1970). Parasites penetrate the gut wall and undergo replication in the hypoderm. Upon host pupation, the parasite forms spores around the scales of the developing butterfly, and infected butterflies emerge covered with dormant spores on the outsides of their bodies. Parasite replication is intense, with single spores giving rise to millions of offspring on the adult butterfly (De Roode et al. 2007). Because higher parasite loads reduce host fitness (De Roode et al. 2007, 2008b, 2009; De Roode \& Altizer 2010), there should be strong selection for host mechanisms that reduce parasite burdens and disease symptoms. Second, monarchs form a tight association with their milkweed larval host plants, which can dramatically alter parasite load and virulence. In a previous study, parasite reproduction was strongly reduced on Asclepias curassavica as compared to Asclepias incarnata, resulting in a strong alleviation of fitness loss (De Roode et al. 2008a). This study also suggested that the anti-parasitic effects of $A$. curassavica could be due to their much higher concentrations of cardenolides, secondary chemicals that milkweeds use against their natural enemies (Malcolm 1991). Thus, there is high potential for medication in this system, because infected monarchs able to preferentially use anti-parasitic host plants would reduce parasite burdens and alleviate their fitness loss.
We tested two predictions: (i) infected monarch larvae do not have the ability to preferentially consume anti-parasitic milkweed species; but (ii) infected adult female monarchs do preferentially lay their eggs on anti-parasitic milkweeds. These predictions are based on the fact that walking larvae are much less able to choose among milkweed species in the wild than are flying adults. Most milkweed species form large, low-density and low-species diversity patches under field conditions (Zalucki et al. 1990), such that monarch larvae have limited choice among milkweed species in nature. In contrast, adult females visit different patches of milkweeds to oviposit (Ladner \& Altizer 2005), and have the ability to encounter and utilize different species.

\section{METHODS}

\section{Milkweeds, monarchs and parasites}

We provided monarchs a choice between $A$. incarnata and A. curassavica, two milkweed species that occur in North America and that differ markedly in their cardenolide concentrations. Plants were grown from seeds obtained from Butterfly Encounters, San Ramon, USA. Monarchs were lab-reared grand-progeny of wild monarchs caught in Pismo Beach, CA, in December 2008. Although A. incarnata and $A$. curassavica are not the most abundant milkweeds in North America, they both occur in the geographic range inhabited by western North American monarchs (http:// plants.usda.gov/java/profile?symbol=ASCLE); thus monarchs used in this study can encounter both species in the wild. Moreover, because host plant selection and host plant effects on infection seem to be mainly mediated by chemicals (e.g. Haribal \& Renwick 1998; De Roode et al. 2008a), the choice of milkweed species is less important than their chemistry differences. In experiments 1 and 2, monarchs were reared on Asclepias tuberosa instead of A. incarnata or $A$. curassavica to avoid potential maternal effects or a potential influence of natal milkweed species on milkweed feeding and/or oviposition preference. The parasite clone denoted C1C10-P2-1 and used in experiments 1 and 2 was derived from an infected butterfly collected in Pismo Beach in 2005.

\section{Experimental design}

Experiment 1: Larval feeding choice (June 2009)

Upon hatching, larvae were transferred into $0.36 \mathrm{~L}$ containers with fresh leaves from $A$. tuberosa as a source of food. Initially, 64 larvae from four non-inbred half-sib monarch families were assigned to an infected group ( $n=32$; eight larvae from each family) or an uninfected control group $(n=32)$. Two days after hatching, when larvae had reached the second instar, they were transferred to individual $10 \mathrm{~cm}$ 
Petri dishes for parasite inoculation. Dishes contained moist filter paper and one $0.8 \mathrm{~cm}$ diameter leaf disc of $A$. tuberosa with 10 parasite spores. Control individuals received leaf discs without parasites. Once larvae had completely eaten their milkweed pieces - and hence ingested the parasite inoculum - they were transferred to individual $0.94 \mathrm{~L}$ containers with meshed lids. Larvae were kept in a climatecontrolled chamber at $26{ }^{\circ} \mathrm{C}$ and a L16:D8 light cycle.

Containers were lined with moist filter paper and caterpillars provided with a choice between two piles of $2.54 \mathrm{~cm}$ diameter leaf discs, consisting of either $A$. incarnata or $A$. curassavica. Larvae received an increasing number of leaf discs as they matured. On day 4 post-inoculation (i.e. day 6 post-hatching), we started using leaf rectangles $(7.5 \times 1.5 \mathrm{~cm})$ instead of discs as the latter were too small to provide the larvae with enough food. Daily, uneaten leaf matter (ULM) from the piles was collected, dried and weighed, and replaced by fresh milkweed. Untouched leaf discs or rectangles (with average dried weight $X$ ) were also removed, dried and weighed. Special care was taken to provide the larvae with more food than needed to correctly assess the feeding preference. The amount of $A$. incarnata consumed $(I)$ was estimated by subtracting $\mathrm{ULM}_{\text {incarnata }}$ from the product of the average $X_{\text {incarnata }}$ and the number of leaf discs or rectangles provided. In the same way, the amount of $A$. curassavica $(C)$ consumed was estimated by subtracting $\mathrm{ULM}_{\text {curassavica }}$ from the product of $X_{\text {curassavica }}$ and the number of leaf discs/rectangles provided. Thus, for each caterpillar, the proportion of the total diet that consisted of A. curassavica during larval development was: $\sum C / \sum(C+I)$. Throughout the experiment, each caterpillar received milkweed from several plants.

\section{Experiment 2: Adult oviposition choice (July 2009)}

Initially, 200 larvae from five different half-sib families were randomly assigned to an infected group $(n=100)$ or an uninfected control group $(n=100)$. Parasite inoculation was performed as described in experiment 1. After inoculation, larvae from both infected and control groups were reared individually in $1.34 \mathrm{~L}$ tubes with shoots of A. tuberosa. The containers were checked daily and larvae provided with fresh cuttings of $A$. tuberosa as needed until pupation. Once monarchs had been in the pupal stage for 6 days (an average of 3 days before eclosion), they were transferred to a second laboratory (maintained at the same temperature and light cycle) to avoid risk of parasite contamination of the larval rearing laboratory by emerging infected adults. Pupae were scored for parasite infection using discolouration of the pupal case on a scale of $0-5$, with 0 being uninfected (no dark patches under the pupal integument) and 5 heavily infected (dark patches forming under the majority of pupal integuments). Pupal scores are highly correlated with actual numbers of parasite spores on adult butterflies (De Roode et al. 2008b, 2009). After eclosion, host sex was determined, and clear tape discs $(2.54 \mathrm{~cm}$ diameter $)$ were pressed onto the abdomens of uninoculated male and female butterflies and examined under a dissecting microscope at $60 \times$ to verify the absence of parasite spores: all individuals in the uninfected group were parasite-free. Males that emerged from the infected group were not used for the experiment.

Three days after eclosion, 49 infected females were transferred to one of five mating cages $\left(0.6 \mathrm{~m}^{3}\right)$ and 43 control female monarchs to one of five other mating cages. Uninfected and genetically unrelated males were added to these cages to serve as mating partners. Cages were checked twice daily, and mating pairs transferred to either the 'infected' or 'control' holding cage. After pairs dissociated, males were returned to their original mating cages. Mated females were left in their new cage and supplied with ad libitum 10\% honey water solution, until they were tested for oviposition preference.

Twenty-seven infected females out of 49 and 14 uninfected females out of 43 did not mate or lay eggs $\left(x^{2}=3.84\right.$, d.f. $\left.=1, P=0.0499\right)$, thus confirming the cost of infection on mating success (De Roode et al. 2009). Oviposition preference tests were performed on the remaining 22 infected and 29 uninfected females. Five $7.1 \mathrm{~m}^{3}$ oviposition cages $(3.1 \mathrm{~m}$ length, $1.8 \mathrm{~m}$ height and $1.27 \mathrm{~m}$ width) were set up in a greenhouse. In each cage, a potted $A$. curassavica and a potted $A$. incarnata plant were randomly placed at the front or the back of the cage. All plants used were 5-6 months old, had been grown under a long photoperiod (L14:D10), were between 40 and $50 \mathrm{~cm}$ in height, and none had yet flowered, set seed or showed signs of senescence. Infected and uninfected females were picked from their respective holding cages and assigned randomly to one of the five oviposition cages. To obtain an estimate of the consistency of their oviposition behaviour, females were tested twice on different days.

Females were released individually into the cages for a period of $2 \mathrm{~h}$, and the number of eggs laid on each of the two plant species was recorded. To account for differences in oviposition caused by variation in the size or biomass of milkweeds, plants were cut at the base of the stem and weighed to the nearest $0.001 \mathrm{~g}$. The leaves of each plant were removed from the stems, oven-dried and weighed. Mean fresh weights $( \pm$ SE) of plants were $14.71( \pm 0.44) \mathrm{g}$ and $25.09( \pm 0.55) \mathrm{g}$ for $A$. incarnata and $A$. curassavica respectively. Mean dry leaf weights were $1.18( \pm 0.04) \mathrm{g}$ and $1.40( \pm 0.04) \mathrm{g}$ for $A$. incarnata and $A$. curassavica respectively. The difference in fresh weights between species $(t=14.8$, $P<0.01$, d.f. $=180)$ was mostly due to the much thicker stems of $A$. curassavica plants. In terms of leaf biomass available for oviposition, $A$. curassavica had a higher biomass than $A$. incarnata $(t=4, P<0.01$, d.f. $=192)$. 
Experiment 3: Host plant effects on monarch and parasite fitness (August 2009)

Monarch larvae belonging to the same genetic lineages and generation as those oviposited in experiment 2 were reared on $A$. incarnata or $A$. curassavica plants, left uninfected (controls) or infected with one of 10 genetically different parasite clones (from four different monarch populations). Parasite reproduction was assessed using the pupal scoring method as described previously. Upon adult eclosion, monarchs were stored at $12{ }^{\circ} \mathrm{C}$ without food access, and the day that they died was recorded. Adult lifespan was calculated and used as a measure of fitness. This measure provides a combined index of adult monarch lifespan which correlates with life time reproductive success (De Roode et al. 2008b, 2009) - and starvation resistance, and responds to parasite infection in a similar way as lifespan under more natural conditions (De Roode et al. 2009). This measure does not provide the full fitness effects of parasitism, which reduces survival, mating ability and lifetime fecundity as well as adult longevity; all these measures correlate negatively with increasing parasite spore loads (De Roode et al. 2008b, 2009). For each host plant-byparasite combination, 30 larvae were inoculated, resulting in a total of 300 larvae. Because some larvae died, and others failed to consume their inocula, the final analysis was restricted to the following numbers of monarchs: lifespan, 130 A. curassavica and 131 A. incarnata; spore load, 131 A. curassavica and 133 A. incarnata. Lifespan of control animals was calculated on the basis of 19 individuals each for $A$. curassavica and $A$. incarnata.

\section{Analysis of cardenolide concentrations in Asclepias foliage}

Milkweed plants for the three experiments described previously were derived from a single batch of purchased seed of each species; genetic relatedness within species was unknown. To explore variation in the cardenolide chemistry of plants from this batch of seed, we assayed plants from each milkweed species from the stock plants grown to run our experiments; the plants sampled for chemistry were not used directly in the experimental work to avoid potential effects of experimental treatment on cardenolide induction (a)

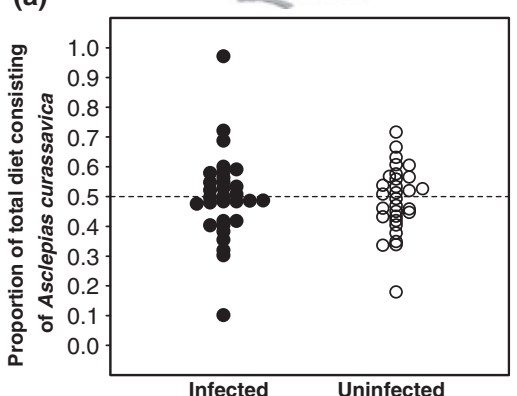

(c)

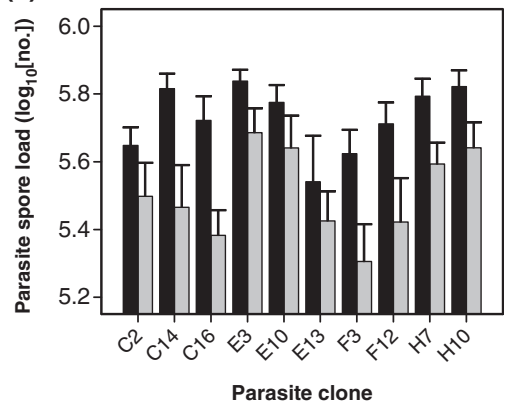

(b)

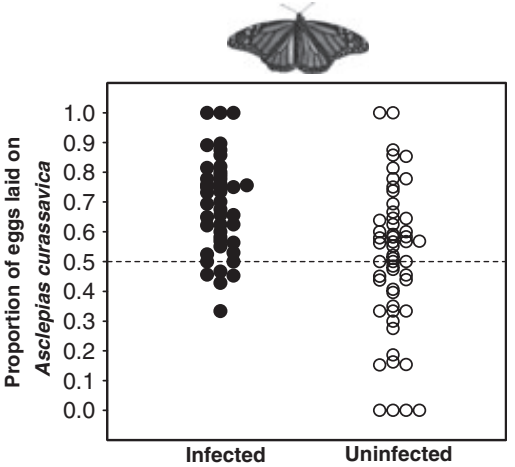

(d)

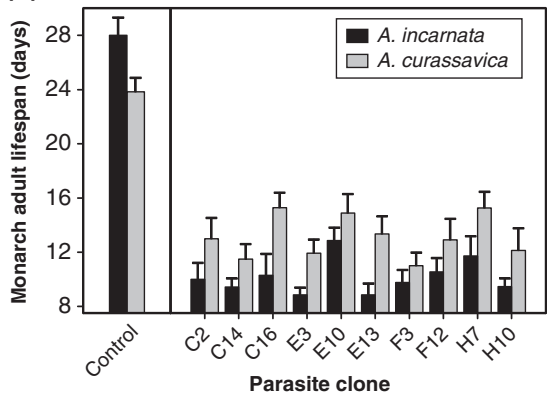

Figure 1 Trans-generational medication in the monarch butterfly. (a) Proportion of the total diet that consisted of Asclepias curassavica for infected $(n=31)$ and uninfected $(n=30)$ caterpillars. (b) Proportion of eggs laid on $A$. curassavica by infected ( $n=44$ observations: 22 females tested twice) and uninfected ( $n=54$ observations: 29 females tested twice; four females laid no eggs during one trial) females. Infected females laid $67 \%$ of their eggs on $A$. curassavica, but uninfected females showed no consistent preference for either species and laid on average $52 \%$ of their eggs on $A$. curassavica. (c) Parasite spore loads of infected monarchs reared on Asclepias incarnata $(n=133)$ or A. curassavica $(n=131)$. Data shown are mean +1 SE. (d) Adult life span of uninfected (control) and infected monarchs reared on A. incarnata ( $n=19$ control and 131 infected) or A. curassavica $(n=19$ control and 130 infected). Data shown are mean $+1 \mathrm{SE}$. 
(Malcolm \& Zalucki 1996). They represent a random subsample of the stock plants used in our three experiments. Foliar cardenolide concentrations were estimated using established methods (Malcolm \& Zalucki 1996; De Roode et al. 2008a). In July 2009, we collected fresh leaf material directly into methanol (24 and 34 individual plants for $A$. curassavica and $A$. incarnata respectively). Samples were homogenized and the extracts analysed using reverse phase high-performance liquid chromatography. Peaks were detected by diode array at $218 \mathrm{~nm}$, and absorbance spectra were recorded from 200 to $300 \mathrm{~nm}$ with digitoxin as an internal standard. Peaks with symmetrical absorption maxima between 217 and $222 \mathrm{~nm}$ were recorded as cardenolides (Malcolm \& Zalucki 1996). We detected a total of 14 different cardenolide peaks, 2 in $A$. incarnata and 13 in $A$. curassavica (they shared one cardenolide peak in common: P1; Fig. 2). Concentrations of each peak were estimated relative to the internal standard and total cardenolide concentration was calculated as the sum of all individual cardenolide peaks.

In addition to analysing the cardenolide concentrations of plants derived from these experiments, we also analysed cardenolides from 29 A. incarnata and 30 A. curassavica plants used in a previous experiment carried out in June 2008. This experiment was similar to experiment 3, except that only one parasite genotype was used to infect monarchs. In this particular experiment, individual caterpillars were fed individually tracked milkweed plants, allowing us to test for associations between the concentrations of particular cardenolides and the parasite spore load produced on monarchs feeding on these plants (Fig. 3).

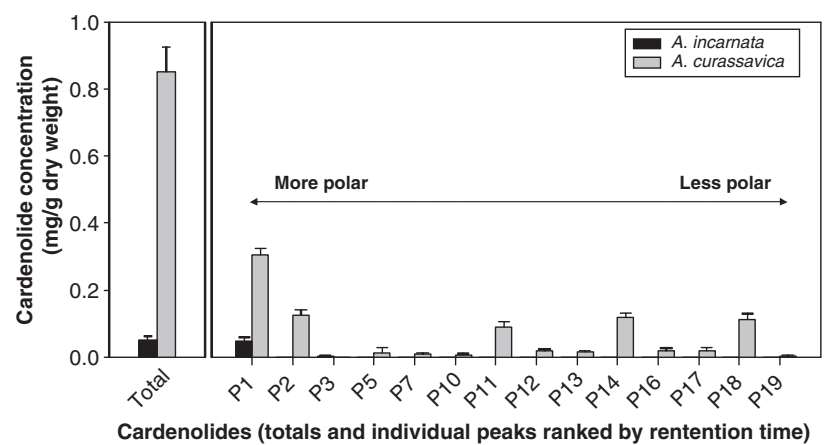

Figure 2 Cardenolide concentrations in the foliage of Asclepias curassavica and Asclepias incarnata, used in experiments 1-3. Concentrations are averages from 34 (A. incarnata) and 24 (A. curassavica) individual plants. Cardenolide diversity and total concentration are higher in $A$. curassavica than in $A$. incarnata. Peak numbers refer to a library of $A$. curassavica peaks developed by $\mathrm{MDH}$ and separated by retention times.

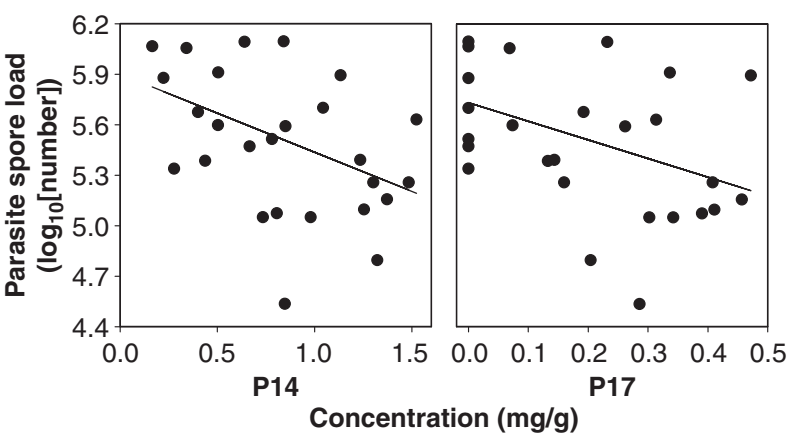

Figure 3 Relationships between the concentration of two nonpolar cardenolides (peaks 14 and 17) in 27 Asclepias curassavica plants and the spore loads on the monarchs fed on these plants.

\section{Statistical analysis}

All analyses were carried out in $\mathrm{R}$ version 2.7.1 and statistical significance was assessed by removing model terms followed by model comparison (Crawley 2002): only terms for which removal significantly $(P<0.05)$ reduced the explanatory power of the model were retained in the minimal model.

\section{Experiment 1}

The proportion of total diet that consisted of $A$. curassavica, and overall food intake, were analysed using multi-way ANOVA. Models were checked for homogeneity of variance by using the Fligner-Killeen tests (Crawley 2002). Full models included infection treatment, monarch sex and family as explanatory variables, and interactions between them.

\section{Experiment 2}

Logistic regression by Generalized Linear Mixed Models (GLMM, binomial errors, logit link) was used to investigate the effect of infection on the proportion of eggs laid on A. curassavica. The influence of several other explanatory variables was investigated by including these in the binomial models: plant biomass (i.e. dry mass and fresh mass), temperature, position of the plants in the cage, and monarch family. As ovipositing females were tested twice, the model was fitted by specifying infection, difference of plant biomass, temperature, position of the plants, cage and monarch family as fixed effects and female ID as a random effect (Crawley 2002). The total number of eggs laid was analysed using multi-way ANOvA. ANCOVA was used to analyse the relationship between the proportion of eggs laid on $A$. curassavica on the first and second trial; this analysis was carried out using untransformed proportions, as model residuals were normally distributed and had homogeneic variance. 


\section{Experiment 3}

ANOVA was used to test for an effect of host plant species, parasite genotype and their interaction on parasite spore load and host adult lifespan. Lifespan was also analysed for uninfected control animals.

\section{Cardenolide concentrations}

Total cardenolide concentrations were compared between species by one-way ANOVA. Relationships between parasite spore load and cardenolide concentration were analysed using linear regression. Concentrations were log-transformed before analysis to meet assumptions of homogeneity of variance.

\section{RESULTS}

\section{Experiment 1: Larval feeding choice (June 2009)}

Infected and uninfected larvae ate a similar amount of milkweed over their entire larval development (mean \pm SE: $1155 \pm 45$ and $1245 \pm 54 \mathrm{mg}$ dry weight respectively), indicating that there was no effect of parasitism on overall food intake $\left(F_{1,53}=2.9, P=0.09\right)$. We found an effect of monarch lineage on overall food intake during larval development, with individuals from a family denoted $\mathrm{P}$ eating more than individuals from the other three families $(F=14.5, P<0.01)$.

There was no difference in the relative diet constitution of infected and uninfected individuals, with $A$. curassavica constituting $50 \%$ of the consumed diet of infected individuals and $49 \%$ of the diet of control individuals $\left(F_{1,59}=0.09, P=0.76\right.$; Fig. 1a). These results indicate that infected larvae are unable to self-medicate, thus supporting prediction (i). There was no effect of host family, sex or interactions between these factors on the proportion of total diet that consisted of $A$. curassavica. A marginally significant interaction between infection and host family was found with the individuals from a family denoted $\mathrm{S}$ eating more when uninfected $(F=3$, $P=0.04)$.

\section{Experiment 2: Adult oviposition choice (July 2009)}

The numbers of eggs laid by individual infected and uninfected females were not statistically different (mean \pm SE: $47.64 \pm 4.9$ and $47.59 \pm 5.1$ respectively; $\left.F_{1,89}=0.0004, P=0.99\right)$. However, infected females laid a significantly higher proportion of their eggs on the antiparasitic $A$. curassavica than did uninfected females [Fig. 1b; GLMM, odds ratio $(\mathrm{OR})=2.28 ; 95 \%$ confidence interval $(\mathrm{CI})=(1.87,2.68) ; P<0.001]$. Individual females were generally consistent in their oviposition choice on consecutive trials, such that females that preferred $A$. curassavica on the first trial also preferred this species on the second trial $\left(F_{1,45}=9.78, P=0.003\right)$. These results support prediction (ii) in that infected females have a strong oviposition preference for the plant species that will alleviate the disease symptoms of their offspring.

There was no significant effect of infection intensity (parasite spore load) on the oviposition preference of infected females $\left(F_{1,36}=0.003, P=0.95\right)$, suggesting a qualitative rather than a quantitative plastic adjustment of oviposition behaviour in response to parasitism. There was a small effect of monarch family lineage $\mathrm{O}$ : $\mathrm{OR}=2.27$; $\mathrm{CI}=(1.57,2.98) ; P=0.02$; lineage $\mathrm{F}: \mathrm{OR}=2.2 ; \mathrm{CI}=(1.4$, $2.9) ; P=0.04$ ] and cages [cage $\mathrm{C}: \mathrm{OR}=1.45 ; \mathrm{CI}=(1.21$, 1.68); $P<0.01$; cage $\mathrm{D}: \mathrm{OR}=1.88 ; \mathrm{CI}=(1.49,2.27)$; $P<0.01]$ on the proportion of eggs laid on $A$. curassavica. No effects of plant biomass, temperature or position of the plants were found. There was also no effect of monarch family, temperature, cage, position of the plants or plant biomass on the total number of eggs laid on either plant species. To further investigate the effects of plant biomass on oviposition behaviour, we reanalysed our data using the number of eggs per gram of foliage instead of the number of eggs laid per plant individual. This analysis yielded the same outcomes with infected females exhibiting a strong preference for $A$. curassavica, while uninfected counterparts displayed no preference [respectively, $\mathrm{OR}=2.23$; $\mathrm{CI}=(2.01,2.43) ; P<0.001$ and $\mathrm{OR}=1.02 ; \mathrm{CI}=(0.67$, 1.37); $P=0.9]$. Thus, the higher biomass of $A$. curassavica cannot explain the oviposition preference for this species of infected females.

\section{Experiment 3: Host plant effects on monarch and parasite fitness (August 2009)}

All tested parasite genotypes produced lower spore loads on monarchs reared on $A$. curassavica than those reared on A. incarnata (Fig. 1c; $F_{1,253}=38.1, P<0.001$ ). Consequently, infected monarchs reared on $A$. curassavica had longer life spans than those reared on $A$. incarnata (Fig. 1d; $\left.F_{1,250}=32.5, P<0.001\right)$. Importantly, although parasite genotypes varied in the spore loads they produced $\left(F_{9,253}=3.25, P<0.001\right)$ and the lifespan reduction that they caused $\left(F_{9,250}=2.55, P=0.008\right)$, there were no host plant $\times$ parasite genotype interactions $\left(F_{9,244}=0.63\right.$, $P=0.77 ; \quad F_{9,241}=0.49, P=0.88$ for spore load and lifespan respectively) demonstrating that $A$. curassavica consistently reduces parasite growth and increases host lifespan, irrespective of parasite genotype. In contrast, uninfected individuals lived longer when reared on A. incarnata (Fig. 1d; $F_{1,250}=32.5, P<0.001$ ) indicating a cost of feeding on $A$. curassavica for this particular fitness measure. Male and female monarchs did not differ in lifespan. 


\section{Analysis of cardenolide concentrations in Asclepias foliage}

We found both qualitative and quantitative differences in cardenolides between the two host plant species used here (Fig. 2). The mean total cardenolide concentration was 17 -fold higher in $A$. curassavica than in $A$. incarnata $\left(F_{1,56}=158, P<0.001\right)$. In addition, $A$. curassavica contained 13 distinct cardenolides types, whereas $A$. incarnata contained only two (P1 and P3; Fig. 2). Finally, the concentrations of two specific non-polar cardenolides from $A$. curassavica were negatively associated with parasite spore load (Fig. 3; peak 14: $F_{1,25}=5.41, P=0.028$; peak 17: $\left.F_{1,25}=4.86, P=0.037\right)$. There were no such relationships with overall concentrations of cardenolides in either A. incarnata or A. curassavica, suggesting that non-polar cardenolides may play a role in mediating the anti-parasitic effects of $A$. curassavica.

\section{DISCUSSION}

Our results support the four criteria that should be met for a behaviour to qualify as therapeutic medication: (1) infected monarchs show an oviposition preference for $A$. curassavica, whereas uninfected monarchs do not; (2) monarch larvae that feed on $A$. curassavica experience lower parasite burdens, and hence experience an alleviation of the fitness costs induced by the parasite; (3) these anti-parasitic benefits come at a cost with reduced longevity of uninfected caterpillars fed on $A$. curassavica; (4) the parasite does not benefit from reduced spore production, as $A$. curassavica reduces parasite spore load (Fig. 1c) to below the level at which parasite fitness is maximized [i.e. $\log _{10}$ (sporeload) of c. 5.7] (De Roode et al. 2008b).

Infected adult butterflies have no known way to cure themselves and are also not able to prevent the transmission of their parasites to their offspring. However, our results demonstrate that they can increase their inclusive fitness by laying their eggs on plants that will alleviate disease in their offspring. Other studies have shown that Spodoptera caterpillars can offset protein costs of pathogen resistance by self-regulating their nutritional intake (Lee et al. 2006; Povey et al. 2009) and that parasitoid-infected arctiid caterpillars can increase the intake of anti-parasitic chemicals from their diet (Singer et al. 2009). Our results complement and extend these self-medication studies to show that animals may also engage in trans-generational medicating behaviour.

Our results provide further support that kin selection can be crucial in driving the evolution of animal behaviours in the wild (Hamilton 1964; Grafen 1984). From a genetic point of view, a gene that gives rise to this behaviour enhances its survival in the population by increasing the fitness of the gene carrier's offspring. This medicating behaviour thereby resembles the maternal transfer of antibodies to offspring in mammals and birds, which also increases inclusive fitness (Hasselquist \& Nilsson 2009).

The fact that we observed a lifespan cost of the antiparasitic plant species for uninfected monarchs is perhaps surprising, as uninfected monarchs did not have an oviposition preference for A. incarnata (Fig. 1b). However, locating optimal host plants is likely costly in nature, where butterflies may need to fly long distances to locate milkweed patches. If locating costs outweigh the fitness costs associated with using non-preferred plants, uninfected females may not have striking oviposition preferences. In contrast, the preference for $A$. curassavica among infected monarchs is readily explained because of the reductions in parasite spore load in their offspring, which alleviate not only reductions in lifespan, but also in pre-adult survival, adult mating ability and fecundity (De Roode et al. 2007, 2008b, 2009; De Roode \& Altizer 2010). Our results suggest that the fitness costs of parasite infection strongly outweigh any costs associated with locating preferred host plants.

The precise mechanism of parasite inhibition by milkweed plants is not yet clear, but previous work (De Roode et al. 2008a) has implicated cardenolides as potential antiparasitic agents. Cardenolides are toxic steroids that monarchs sequester in their tissues, making them unpalatable to predators (Brower et al. 1968; Malcolm \& Brower 1989). It is therefore possible that these chemicals also mediate the anti-parasitic effects of milkweed species (De Roode et al. 2008a). Among the plants used for the oviposition and larval performance experiments described here, total cardenolide concentrations were much higher in A. curassavica than in $A$. incarnata. Moreover, $A$. curassavica plants contained many non-polar cardenolides, whose concentrations correlate with parasite spore load. Cardenolides usually act on $\mathrm{Na}+, \mathrm{K}+$-ATPases (Malcolm 1991); although we do not yet know if such proteins occur in O. elektroscirrba, $\mathrm{Na}+, \mathrm{K}+$-ATPases have been found in other eukaryotic protozoan parasites (Felibertt et al. 1995). Thus, our data suggest that cardenolides may play a role in mediating the anti-parasitic effects of milkweeds, but manipulative experiments are needed to confirm this.

Similarly, the proximate mechanism by which infected butterflies alter their oviposition preference is currently unknown. Infection may induce changes in receptors involved in milkweed-seeking behaviour and preference, including the olfactory and contact chemoreceptor sensilla on the legs and antennae of the butterfly (Haribal \& Renwick 1998). Similar mechanisms have been proposed to explain changes in diet preference of infected arctiid larvae (Singer et al. 2009), which show increased responsiveness of taste receptors to protective plant toxins (Bernays \& Singer 2005).

Beyond demonstrating medication, our results support the hypothesis that lepidopterans have evolved the ability to 
lay their eggs on host plants that will increase their offspring's survival (Jaenike 1978; Thompson 1988; Thompson \& Pellmyr 1991; Gripenberg et al. 2010). In the case of monarch butterflies, the factors proposed to influence milkweed preference until now have included host plant diversity, abundance, age, size, larval performance and predation (Ladner \& Altizer 2005). Our study not only demonstrates that parasitism should be added to this list, but may also help explain conflicting results on monarch oviposition preferences for $A$. incarnata and $A$. curassavica in previous studies. For instance, while a study in a mixed stand of these species in South Florida reported a strong oviposition preference for $A$. curassavica (Malcolm \& Brower 1986), a study in northern Florida failed to demonstrate any preferences (Zalucki et al. 1990). These contradictory results could be reconciled knowing that: (1) South Florida is the geographic area where the highest $O$. elektroscirrha prevalence has been reported so far (Altizer et al. 2000); and (2) the monarchs used in the second study emerged in the laboratory and were derived from a population with low parasite prevalence.

Most host-parasite studies focus on genetic interactions between parasites and their hosts (Lambrechts et al. 2006) without considering the effect of the environment (Wolinska \& King 2009). Our results emphasize that environmental conditions can strongly affect parasite virulence and host resistance (Cory \& Hoover 2006), and that hosts can directly alter these selection pressures. This study also reinforces the idea that animal medication may inform human drug discovery and development (Huffman 2003). Specifically, our results suggest that monarch butterflies have evolved the ability to detect and take advantage of antiparasitic substances to combat $O$. elektroscirrha. Identifying these substances may have potential use for humans, who suffer from parasite species - such as Cryptosporidium and Plasmodium spp. - that are related to this monarch-specific parasite (Carreno et al. 1999).

\section{ACKNOWLEDGEMENTS}

We thank J. Huang, C. Lopez, J. Li, H. Li, R. Rarick and E. Sternberg for their help with the experiments, R. Antia, F. Thomas, A. Read, the Emory IHoP club, three referees and M. van Baalen for their comments on the manuscript. This work was funded by Emory University, a SingerPolignac stipend to TL, Emory SURE program studentship to LO, NSF grant DEB-0814340 to MDH and NSF grant DEB-1019746 to JCdR and MH.

\section{REFERENCES}

Altizer, S.M., Oberhauser, K.S. \& Brower, L.P. (2000). Associations between host migration and the prevalence of a protozoan parasite in natural populations of adult monarch butterflies. Ecol. Entomol., 25, 125-139.

Bernays, E.A. \& Singer, M.S. (2005). Taste alteration and endoparasites. Nature, 436, 476-476.

Brower, L.P., Ryerson, W.N., Coppinger, L. \& Glazier, S.C. (1968). Ecological chemistry and the palatability spectrum. Science, 161, 1349-1350.

Carreno, R.A., Martin, D.S. \& Barta, J.R. (1999). Cryptosporidium is more closely related to the gregarines than to coccidia as shown by phylogenetic analysis of apicomplexan parasites inferred using small-subunit ribosomal RNA gene sequences. Parasitol. Res., 85, 899-904.

Castella, G., Chapuisat, M. \& Christe, P. (2008). Prophylaxis with resin in wood ants. Anim. Behav., 75, 1591-1596.

Chapuisat, M., Oppliger, A., Magliano, P. \& Christe, P. (2007). Wood ants use resin to protect themselves against pathogens. Proc. R. Soc. Lond. B-Biol. Sci., 274, 2013-2017.

Christe, P., Oppliger, A., Bancala, F., Castella, G. \& Chapuisat, M. (2003). Evidence for collective medication in ants. Ecol. Lett., 6, 19-22.

Clayton, D.H. \& Wolfe, N.D. (1993). The adaptive significance of self-medication. Trends Ecol. Evol., 8, 60-63.

Combes, C. (2001). Parasitism the Ecology and Evolution of Intimate Interactions. University Press, Chicago.

Cory, J.S. \& Hoover, K. (2006). Plant-mediated effects in insectpathogen interactions. Trends Ecol. Evol., 21, 278-286.

Crawley, M.J. (2002). Statistical Computing: An Introduction to Data Analysis Using S-Plus. John Wiley \& Sons, Chichester.

De Roode, J.C. \& Altizer, S. (2010). Host-parasite genetic interactions and virulence-transmission relationships in natural populations of monarch butterflies. Evolution, 64, 502-514.

De Roode, J.C., Gold, L.R. \& Altizer, S. (2007). Virulence determinants in a natural butterfly-parasite system. Parasitology, 134, 657-668.

De Roode, J.C., Pedersen, A.B., Hunter, M.D. \& Altizer, S. (2008a). Host plant species affects virulence in monarch butterfly parasites. J. Anim. Ecol., 77, 120-126.

De Roode, J.C., Yates, A.J. \& Altizer, S. (2008b). Virulencetransmission trade-offs and population divergence in virulence in a naturally occurring butterfly parasite. Proc. Natl. Acad. Sci. USA, 105, 7489-7494.

De Roode, J.C., Chi, J., Rarick, R.M. \& Altizer, S. (2009). Strength in numbers: high parasite burdens increase transmission of a protozoan parasite of monarch butterflies (Danaus plexippus). Oecologia, 161, 67-75.

Felibertt, P., Bermudez, R., Cervino, V., Dawidowicz, K., Dagger, F., Proverbio, T. et al. (1995). Ouabain-sensitive Na+, K+ATPase in the plasma membrane of Leishmania mexicana. Mol. Biochem. Parasitol., 74, 179-187.

Grafen, A. (1984). Natural selection, kin selection and group selection. In: Behavioural Ecology, 2nd edn. (eds Krebs, J.R. \& Davies, N.B.). Blackwell Scientific Publications, Oxford, pp. 62-84.

Gripenberg, S., Mayhew, P.J., Parnell, M. \& Roslin, T. (2010). A meta-analysis of preference-performance relationships in phytophagous insects. Ecol. Lett., 13, 383-393.

Hamilton, W.D. (1964). Genetical evolution of social behavior I. J. Theor. Biol., 7, 1-16.

Haribal, M. \& Renwick, J.A.A. (1998). Differential postalightment oviposition behavior of monarch butterflies on Asclepias species. J. Insect Behav., 11, 507-538. 
Hart, B.L. (1990). Behavioral adaptations to pathogens and parasites: five strategies. Neurosci. Biobehav. Rev., 14, 273-294.

Hasselquist, D. \& Nilsson, J.-A. (2009). Maternal transfer of antibodies in vertebrates: trans-generational effects on offspring immunity. Philos. Trans. R. Soc. Lond. B Biol. Sci., 364, 51-60.

Huffman, M.A. (2003). Animal self-medication and ethno-medicine: exploration and exploitation of the medicinal properties of plants. Proc. Nutr. Soc., 62, 371-381.

Huffman, M.A. \& Seifu, M. (1989). Observations on the illness and consumption of a possibly medicinal plant Vernonia amygdalina (Del.), by a wild chimpanzee in the Mahale Mountains National Park, Tanzania. Primates, 30, 51-63.

Hutchings, M.R., Athanasiadou, S., Kyriazakis, I. \& Gordon, I.J. (2003). Can animals use foraging behaviour to combat parasites? Proc. Nutr. Soc., 62, 361-370.

Jaenike, J. (1978). On optimal oviposition behaviour by phytophagous insects. Theor. Popul. Biol., 14, 350-356.

Ladner, D.T. \& Altizer, S. (2005). Oviposition preference and larval performance of North American monarch butterflies on four Asclepias species. Entomol. Exp. Appl., 116, 9-20.

Lambrechts, L., Fellous, S. \& Koella, J.C. (2006). Coevolutionary interactions between host and parasite genotypes. Trends Parasitol., 22, 12-16.

Lee, K.P., Cory, J.S., Wilson, K., Raubenheimer, D. \& Simpson, S.J. (2006). Flexible diet choice offsets protein costs of pathogen resistance in a caterpillar. Proc. R. Soc. B-Biol. Sci., 273, $823-829$

Lefèvre, T., Adamo, S.A., Biron, D.G., Misse, D., Hughes, D. \& Thomas, F. (2009). Invasion of the body snatchers: the diversity and evolution of manipulative strategies in host-parasite interactions. Adv. Parasitol., 68, 45-83.

Leong, K.L.H., Yoshimura, M.A. \& Kaya, H.K. (1997). Occurrence of a neogregarine protozoan, Ophryocystis elektroscirrba McLaughlin and Myers, in populations of monarch and queen butterflies. Pan-Pac. Entomol., 73, 49-51.

Lisonbee, L.D., Villalba, J.J., Provenza, F.D. \& Hall, J.O. (2009). Tannins and self-medication: implications for sustainable parasite control in herbivores. Behav. Processes, 82, 184-189.

Lozano, G.A. (1998). Parasitic stress and self-medication in wild animals. Adv. Stud. Behav., 27, 291-317.

Malcolm, S.B. (1991). Cardenolide-mediated interactions between plants and herbivores. In: Herbivores: Their Interactions With Secondary Plant Metabolites (eds Rosenthal, G.A. \& Berenbaum, M.R.). Academic Press, San Diego, pp. 251-296.

Malcolm, S.B. \& Brower, L.P. (1986). Selective oviposition by monarch butterflies (Danaus plexippus L.) in a mixed stand of Asclepias curassavica L. and A. incarnata L. in South Florida. J. Lepidopt. Soc., 40, 255-263.
Malcolm, S.B. \& Brower, L.P. (1989). Evolutionary and ecological implications of cardenolide sequestration in the monarch butterfly. Experientia, 45, 284-295.

Malcolm, S.B. \& Zalucki, M.P. (1996). Milkweed latex and cardenolide induction may resolve the lethal plant defence paradox. Entomol. Exp. Appl., 80, 193-196.

McLaughlin, R.E. \& Myers, J. (1970). Ophryocystis elektroscirrba sp. n., a neogregarine pathogen of monarch butterfly Danaus plexippus (L.) and the Florida queen butterfly D. gilippus berenice Cramer. J. Protozool., 17, 300-305.

Moore, J. (2002). Parasites and the Behavior of Animals. Oxford University Press, Oxford.

Povey, S., Cotter, S.C., Simpson, S.J., Lee, K.P. \& Wilson, K. (2009). Can the protein costs of bacterial resistance be offset by altered feeding behaviour? J. Anim. Ecol., 78, 437-446.

Råberg, L., Sim, D. \& Read, A.F. (2007). Disentangling genetic variation for resistance and tolerance to infectious disease in animals. Science, 318, 318-320.

Rolff, J. \& Siva-Jothy, M.T. (2003). Invertebrate ecological immunology. Science, 301, 472-475.

Singer, M.S., Mace, K.C. \& Bernays, E.A. (2009). Self-medication as adaptive plasticity: increased ingestion of plant toxins by parasitized caterpillars. PLOS ONE, 4, e4796.

Thompson, J.N. (1988). Evolutionary ecology of the relationship between oviposition preference and performance of offspring in phytophagous insects. Entomol. Exp. Appl., 47, 3-14.

Thompson, J.N. \& Pellmyr, O. (1991). Evolution of oviposition behavior and host preference in Lepidoptera. Annu. Rev. Entomol., 36, 65-89.

Windsor, D.A. (1998). Most of the species on Earth are parasites. Int. J. Parasitol., 28, 1939-1941.

Wolinska, J. \& King, K.C. (2009). Environment can alter selection in host-parasite interactions. Trends Parasitol., 25, 236244.

Wrangham, R.W. \& Nishida, T. (1983). Aspilia spp leaves: a puzzle in the feeding behavior of wild chimpanzees. Primates, 24, 276-282.

Zalucki, M.P., Brower, L.P. \& Malcolm, S.B. (1990). Oviposition by Danaus plexippus in relation to cardenolide content of 3 Asclepias species in the southeastern USA. Ecol. Entomol., 15, 231-240.

Editor, Minus van Baalen

Manuscript received 18 August 2010

First decision made 7 September 2010

Manuscript accepted 14 September 2010 